\title{
Controllably local gene delivery mediated by polyelectrolyte multilayer films assembled from gene-loaded nanopolymersomes and hyaluronic acid
}

This article was published in the following Dove Press journal:

International Journal of Nanomedicine

29 October 2014

Number of times this article has been viewed

\author{
Wei Tengl,* \\ Qinmei Wang 2 ,* \\ Ying Chen ${ }^{2}$ \\ Hongzhang Huang' \\ 'Hospital of Stomatology, Institute \\ of Stomatological Research, Guanghua \\ School of Stomatology, Guangzhou, \\ People's Republic of China; ${ }^{2}$ Key \\ Laboratory on Assisted Circulation, \\ Ministry of Health, Cardiovascular \\ Division, First Affiliated Hospital, \\ Sun Yat-sen University, Guangzhou, \\ People's Republic of China \\ *These authors contributed equally \\ to this work
}

\begin{abstract}
To explore a spatiotemporally controllable gene delivery system with high efficiency and safety, polyelectrolyte multilayer (PEM) films were constructed on titanium or quartz substrates via layer-by-layer self-assembly technique by using plasmid deoxyribonucleic acidloaded lipopolysaccharide-amine nanopolymersomes (pNPs) as polycations and hyaluronic acid (HA) as polyanions. pNPs were chosen because they have high transfection efficiency $(>95 \%)$ in mesenchymal stem cells (MSCs) and induce significant angiogenesis in zebrafish in conventional bolus transfection. The assembly process of PEM films was confirmed by analyses of quartz crystal microbalance with dissipation, X-ray photoelectron spectroscopy, infrared, contact angle, and zeta potential along with atomic force microscopy observation. Quartz crystal microbalance with dissipation analysis reveals that this film grows in an exponential mode, pNPs are the main contributor to the film mass, and the film mass can be modulated in a relatively wide range $\left(1.0-29 \mu \mathrm{g} / \mathrm{cm}^{2}\right)$ by adjusting the deposition layer number. Atomic force microscopy observation shows that the assembly leads to the formation of a patterned film with three-dimensional tree-like nanostructure, where the branches are composed of beaded chains (pNP beads are strung on HA molecular chains), and the incorporated pNPs keep structure intact. In vitro release experiment shows that plasmid deoxyribonucleic acid can be gradually released from films over 14 days, and the released plasmid deoxyribonucleic acid exists in a complex form. In vitro cell experiments demonstrate that PEM films can enhance the adhesion and proliferation of MSCs and efficiently transfect MSCs in situ in vitro for at least 4 days. Our results suggest that a ( $\mathrm{pNPs} / \mathrm{HA})_{\mathrm{n}}$ system can mediate efficient transfection in stem cells in a spatially and temporally controllable pattern, highlighting its huge potential in local gene therapy.

Keywords: localized gene delivery, layer-by-layer self-assembly, gene-loaded nanopolymersomes, hyaluronic acid, polyelectrolyte multilayer films, mesenchymal stem cells
\end{abstract}

\section{Introduction}

Substrate-mediated gene delivery can provide spatiotemporal control for localized gene transfection. In this therapeutic strategy, plasmid deoxyribonucleic acid (pDNA) is first immobilized onto a biomaterial surface and then released in a controlled and sustained manner to transfect cells surrounding materials. ${ }^{1-7}$ As compared with the conventional bolus nonviral gene delivery, where cells are transfected by the suspended pDNA/vector complexes in liquid, substrate-mediated gene delivery has many advantages. The first one is its controllability, where gene transfection can be designed to meet clinical needs through controlling the amount of immobilized pDNA and its subsequent release from the substrate surfaces. The second one is its high bioavailability of pDNA due to the high localized concentration of pDNA available to cells around substrates. It has been
Correspondence: Hongzhang Huang Hospital of Stomatology, Sun Yat-sen University, 56 Linyuanxi Road, Guangzhou, Guangdong 510600 , People's Republic of China Tel +862087330757

Email huanghongzhang@tom.com 
demonstrated that only $\sim 20 \%$ of the added pDNA is utilized by cells in bolus delivery, ${ }^{8}$ and to reach a comparable transfection efficiency, the quantity of pDNA needed in substrate-mediated gene delivery is $4.5 \%$ of that in bolus delivery. ${ }^{9}$ The third advantage is it can avoid the unwanted effects from systematic administration, which means higher safety.

Polyelectrolyte multilayer (PEM) films, which can be obtained from alternate deposition of polyanions and polycations in aqueous solutions, are usually used as a reservoir for pDNA in substrate-mediated gene delivery. ${ }^{5,10}$ The release of pDNA can be controlled by adjusting the degradation or disassembly of PEM films under physiological environment. As compared with other modes, DNA-containing PEM films afford numerous potential advantages for pDNA immobilization, such as 1) substrate-independent coating under a mild condition without organic solvents, and 2) easy control over properties of PEM films, including surface chemistry, roughness, stability, degradability, thickness, and mass via changing polyelectrolytes and preparation parameters, and thus realize the protection to pDNA, spatial, and temporal control over pDNA release, promotion in gene transfection, and control over cell responses on substrate surfaces. . $^{3,5,10,11}$

In PEM films, pDNA mostly acts as a polyanion layer $^{3-6,12,13}$ and partially as an adsorbed drug in a complex form (polyplex or lipoplex)., ${ }^{2,5,9,11}$ Both systems can successfully transfect cells in vitro and in vivo, but most of them have relatively low transfection efficiency $(<40 \%)$ due to their own defects. ${ }^{2,5,9}$ When pDNA acts as anionic layers, there are two main drawbacks. The first one is that some released pDNA exists in a naked form. ${ }^{3}$ It is well known that naked pDNA is insufficient in gene transfection due to its poor cellular uptake and rapid degradation by nuclease. ${ }^{14}$ The second drawback is that although the gross mass of polyelectrolytes can be controlled to some extent by adjusting layer numbers in such PEM films, ${ }^{5,10,11}$ how to precisely regulate the mass ratio of polycations to pDNA is unknown. Accordingly, the N/P ratios (nitrogen in vectors/phosphate in pDNA) of complexes formed from released pDNA and polycations cannot be controlled. That is, the N/P ratios of complexes are random. It is well known that the optimal transfection is obtained at optimal N/P ratio in bolus transfection. Therefore, random N/P may lead to low transfection efficiency. Using pDNA as adsorbed drug in the complex form with optimal $\mathrm{N} / \mathrm{P}$ ratios in PEM films can solve the problem of optimal transfection, but its limit in adsorption mass of pDNA will affect its long-time release and transfection.

Using nanocomplexes (polyplexes or lipoplexes) as polycations to construct PEM films may be an alternative strategy to address the aforementioned issues. In this case, the $\mathrm{N} / \mathrm{P}$ ratio, loading mass, and subsequent release behavior of complexes can be easily controlled by changing preparation parameters. To our knowledge, limited studies have been performed in this field to date. Yamauchi et $\mathrm{al}^{15}$ have constructed a PEM film self-assembled from cationic lipoplex (complex of lipofectamine 2000 and pDNA) and anionic naked pDNA on gold substrates. The functionalized gold substrates have been proved to continuously transfect cells seeded on them in vitro, suggesting the potential of this gene delivery system in implantology. Authors deduced from the high transfection efficiency that transfection might be mediated by released lipoplexes but not naked pDNA. Therefore, it is more economic and controllable to choose the other degradable polyanions as anionic layer components. Additionally, the lipoplex may be another limitation due to the low transfection efficiency of cationic lipid in vivo. ${ }^{14}$

Based on the aforementioned developments and limitations in substrate-mediated gene delivery, in this study we used pDNA-loaded lipopolysaccharide-amine (LPSA) nanopolymersomes (pNPs) as polycations, and hyaluronic acid (HA) as a polyanion to build up PEM films. It is worth noting that lipopolysaccharide-amine nanopolymersomes (NPs) are a novel cytosolic delivery vector developed by our group. ${ }^{16}$ LPSA is a degradable, amphiphilic, and amphoteric brush graft copolymer containing anionic oxidized alginate (backbone) and cationic polyethylenimine grafted by hydrophobic cholesterol (side chains), and it can quickly selfassemble into pNPs (diameter of $110 \mathrm{~nm}$ ) in distilled water. As a gene vector, it obtains $>95 \%$ transfection efficiency in mesenchymal stem cells (MSCs) when delivering plasmid encoding enhanced green fluorescence protein DNA ( $p E G F P$ ) in vitro, ${ }^{16}$ and induces significant dose-dependent angiogenesis in zebrafish when delivering plasmid encoding vascular endothelial growth factor DNA $(p V E G F) \cdot{ }^{17} \mathrm{HA}$ is a natural anionic polysaccharide, which can be degraded by hyaluronidase in vivo, and has been widely used in substrate-mediated gene delivery as a polyanion to construct PEM films..$^{2,5,10}$ Therefore, we expect such a film will offer controllable, local, and continuous target proteins produced by cells that are transfected by controllably released complexes of pDNA and NPs. In this paper, we monitor the buildup of PEM films of ( $\mathrm{pNPs} / \mathrm{HA})_{\mathrm{n}}$, explore their growth mechanism, characterize their surface properties, then study the release of pDNA, and, finally, perform a preliminary study on transfection in MSCs in vitro. Considering the homing ability of MSCs to injury and their excellent performance in cell therapy, such PEM films may find wide applications in regenerative medicine. 


\section{Material and methods Materials}

pNPs with hydrodynamic diameter of $\sim 112 \mathrm{~nm}$ at N/P of 60 (optimal for in vitro transfection) were prepared according to our previously published method. ${ }^{16}$ Briefly, LPSA (mass ratio of three blocks, polyethyleneimine:cholesterol:oxidized alginate $=48.5: 11.2: 40.3$ ) was first synthesized by graft copolymerization, then LPSA was directly dissolved in water under stirring to obtain a $2 \mathrm{mg} / \mathrm{mL}$ solution, and pNPs were prepared by vortex mixing of equal volume of LPSA solution and pDNA solution. The plasmid expressing EGFP was purchased from FulenGene (Guangzhou, People's Republic of China), and its amplification and purification followed an established method. ${ }^{16}$ Titanium (Ti) discs with diameter of $10 \mathrm{~mm}$ were purchased from Goodfellow Cambridge Ltd. (USA). The quartz was purchased from Zhongke Optic (Jiangshu, People's Republic of China). HA ( $\left.\mathrm{M}_{\mathrm{w}}, 65 \mathrm{kDa}\right)$ was obtained from Dali Hyaluronic Ccid Co., Ltd., of Liuzhou Chemical Group (Guangxi, People's Republic of China). Sprague Dawley rats were purchased from the Laboratory Animal Center of Sun Yat-sen University (Guangzhou, People's Republic of China). All animal experiments were approved by the Animal Ethics Committee of Sun Yat-sen University and conducted according to Institutional Animal Care and Use Committee Guidelines. The other reagents were purchased from local suppliers. All reagents were used as received without further purification.

\section{Pretreatment of substrates}

Ti (titanium) and quartz substrates were used in this study. Ti was chosen because it has been applied widely in the implantology field but its surface is bioinert, and a genefunctionalized surface can significantly improve bioactivity for more efficient therapy. Ti discs were polished using silicon carbide sand papers and ultrasonically washed for 15 minutes with acetone, ethanol, and double distilled water, in turn. Then, for subsequent assembly, a thin sodiumtitanate-hydrogel layer with negative charges was introduced into their surfaces via alkaline heat treatment according to the reported method. ${ }^{18}$ Ti substrates were mainly used in assays of X-ray photoelectron spectroscopy (XPS), infrared, surface contact angle, pDNA release, and cell experiments.

Quartz substrates were used for those assays requiring transparent or relatively smooth interfaces. The quartz glass was soaked in a piranha solution $\left(\mathrm{H}_{2} \mathrm{SO}_{4}: \mathrm{H}_{2} \mathrm{O}_{2}=7: 3\right.$ by volume) at $\sim 50^{\circ} \mathrm{C}$ for 10 minutes, ultrasonically washed in Milli-Q water for 10 minutes, and blown dry with a stream of $\mathrm{N}_{2}$, in turn. Such treatment not only cleans the surface but also introduces large anions for subsequent deposition of cationic layer.

\section{Self-assembly of PEM films from cationic pNPs and anionic HA on pretreated substrates}

Amounts of $1 \mathrm{mg} / \mathrm{mL}$ pNPs $(\mathrm{N} / \mathrm{P}=60)$ and $1 \mathrm{mg} / \mathrm{mL} \mathrm{HA}$ in $0.15 \mathrm{~mol} / \mathrm{L} \mathrm{NaCl}$ aqueous solution were used in PEM film buildup in this study unless otherwise specified. At room temperature, pretreated substrates were alternately immersed in polyelectrolyte solutions (pNPs or HA) for 10 minutes and rinsed with $\mathrm{NaCl}$ solution for 5 minutes three times after each deposition of polyelectrolytes. It should be noted that $1 \mathrm{mg} / \mathrm{mL}$ of $\mathrm{HA}$ for 10 minutes of incubation is safe for film construction because by gel electrophoresis experiment we demonstrated that HA could not dissociate pNPs during 10-minute coincubation even at high concentration of $200 \mu \mathrm{g} / \mathrm{mL}$. A successive deposition of pNPs and HA on substrate surfaces is defined as one cycle of assembly ([pNPs/HA $\left.]_{1}, \mathrm{C}_{1}\right)$. In this study, two types of PEM films, $(\mathrm{pNPs} / \mathrm{HA})_{4.5}\left(\mathrm{C}_{4.5}\right)$ and $(\mathrm{pNPs} / \mathrm{HA})_{5}\left(\mathrm{C}_{5}\right)$, were prepared, which provides two outermost surfaces - the former pNPs and the latter HA.

\section{Analysis of film self-assembly by quartz crystal microbalance with dissipation}

The construction of films was monitored in real time by a quartz crystal microbalance with dissipation (QCM-D) (D300, Q-Sense AB, Västra Frölunda, Sweden). During self-assembly, the changes in frequency (f) and dissipation (D) with time were recorded. The measurement method has been reported in detail elsewhere. ${ }^{19-22}$ Briefly, the gold-coated resonator (Q-Sense AB) was cleaned with a piranha solution, rinsed with water, blown dry with nitrogen gas, and placed in a QCM holder. Then, at $25^{\circ} \mathrm{C} \pm 0.02^{\circ} \mathrm{C}, \mathrm{NaCl}$ and polyelectrolyte solutions (pNPs or HA) were allowed to alternately flow through the QCM chamber at $50 \mu \mathrm{L} / \mathrm{min}$ until frequency was unchanged. The measurements were taken continuously at the fundamental, third, fifth, and seventh overtones $(5,15,25$, and $35 \mathrm{MHz})$. It is known that $\Delta \mathrm{f}$ is proportional to the mass, and $\Delta \mathrm{D}$ reflects the thickness and viscoelasticity of deposited layer on the resonator. Herein, the thickness and mass of the layer during assembly were fitted based on a Voigt model with the Q-Tools software from Q-Sense AB, following the reported method. ${ }^{19-22}$

\section{Surface characterization on PEM-deposited substrates}

All samples were air dried before measurements, and all analyses were performed at $25^{\circ} \mathrm{C}$. The surface chemical structure 
of modified Ti substrates was analyzed by Fourier transform infrared (FTIR) spectrometer (EQUINOX 55; Bruker Optik $\mathrm{GmbH}$, Ettlingen, Germany), and their surface elemental compositions were determined by XPS (ESCALab250, Thermo Fisher Scientific Inc., Waltham, MA, USA) under a pressure of $\sim 2 \times 10^{-9}$ mbar with a monochromatic Al K $\alpha$ radiation X-ray source $(1486.6 \mathrm{eV}, 15 \mathrm{kV}, 150 \mathrm{~W})$. Charge referencing for XPS was performed by setting the main $\mathrm{C} 1 \mathrm{~s}$ (C-C/C-H) component at $284.8 \mathrm{eV}$. Their contact angles with a pure water droplet were measured using a contact angle meter (PHOENIX 150; Surface Electro Optics, Suwon City, Gyunggido, Korea), and 15 measurements per group (five different sites/sample $\times$ three samples) were averaged. The following characterization was performed on modified quartz-glass substrates. Their zeta potentials were determined by a zeta potential and nanoparticle size analyzer (Delsa ${ }^{\mathrm{TM}}$ Nano C, Beckman Coulter, Brea, CA, USA). Their surface topography and roughness were observed in air using atomic force microscopy (AFM) (Nanoscope V, Santa Barbara, CA, USA) with a standard silicon cantilever in a tapping mode.

\section{Release of pDNA from PEM films}

PEM film-functionalized Ti was immersed in $2 \mathrm{~mL}$ of phosphate-buffered saline (PBS, pH 7.4) at $37^{\circ} \mathrm{C}$. At designed time points, $1 \mathrm{~mL}$ of the supernatant was collected, and 1 $\mathrm{mL}$ of fresh PBS was supplemented. The amount of released pDNA in the supernatants was measured by ultraviolet-visible spectrophotometer (Nanodrop 2000, Thermo Scientific) after concentration treatment by centrifugal ultrafiltration method. It should be noted that the existence form of pDNA (naked or loaded in NPs) nearly has no effects on its ultraviolet absorbance (data not shown). The gross mass of pDNA incorporated in PEM films was determined by measuring the concentration of pDNA in pNP solutions before and after deposition along with in rinsing solutions using the same method. Additionally, the existence form of released pDNA in supernatants was investigated as follows. Briefly, the concentrated supernatant was first incubated with heparin (final concentration, $20 \mathrm{mg} / \mathrm{mL}$ ) for 2 hours at room temperature to completely dissociate pDNA from pNPs. ${ }^{17}$ Then, supernatants with or without heparin-dissociation treatment were analyzed by gel electrophoresis.

\section{Adhesion and proliferation of bone marrow MSCs on PEM-deposited Ti substrates}

MSCs were obtained from neonatal Sprague Dawley rats at 1-3 days of age, and their isolation, purification, culture, and passage were performed according to the standard method. ${ }^{23}$ The adhesion of MSCs on PEM-deposited Ti discs was assessed according to the reported method using AlamarBlue assay. ${ }^{24}$ Briefly, MSCs (passages 3-7) were seeded in 24-well plates with Ti at a density of $2.5 \times 10^{4} / \mathrm{cm}^{2}$ and cultured for 15 minutes in $1 \mathrm{~mL}$ complete media (Hyclone DMEM/F12 $1: 1$ supplemented with $10 \%$ fetal bovine serum, $100 \mu \mathrm{g} / \mathrm{mL}$ streptomycin, and $100 \mathrm{U} / \mathrm{mL}$ penicillin). Then, the plate was slightly agitated for 3 minutes, and the supernatants were transferred to new empty 24-well plates. Fresh complete medium was added into the original plates, and all plates were routinely cultured for additional 2 hours. Thereafter, $10 \%$ AlamarBlue (Invitrogen) was added into the culture medium, and all cells were continuously cultured for 3 hours. After 3 hours, the absorbance of supernatants in all wells at $570 \mathrm{~nm}$ and $600 \mathrm{~nm}$ was measured on an enzyme-linked immunosorbent assay plate reader (Model 550, BioRad), and cells in original plates were fixed and stained by 4',6diamidino-2-phenylindole for visual observation using fluorescent microscopy. The cell adhesion ability was expressed as the percentage of the absorbance from the cells attached in 15 minutes relative to the absorbance from the seeded cells (cells attached + cells in supernatant). The bare Ti and alkaline heat-treated $\mathrm{Ti}$ were used as controls.

For proliferation assessment, MSCs were seeded at a density of $1.0 \times 10^{4} / \mathrm{cm}^{2}$ and cultured routinely for designed time intervals. At each time point, the absorbance of each well was determined using AlamarBlue assay as mentioned. Simultaneously, cells were stained with rhodamine phalloidin (Biotium Inc., USA) to fluorescently label F-actin of the cytoskeleton following the manufacturer's protocol. The stained cells were imaged using fluorescence microscopy (IX 71, Olympus, Japan).

\section{Transfection in MSCs in vitro}

In vitro transfection activity of PEM films was evaluated by using MSCs as target cells and pEGFP as a target gene. MSCs were plated on functionalized Ti in 24-well plates (five wells per group) and cultured as described. At designed time points, some cells were observed under a fluorescence microscope, and the others were lysed and fixed for determination of transfection efficiency (percentage of MSCs expressing green fluorescence protein [GFP]) using a Coulter flow cytometer (Becton Dickinson). ${ }^{16} \mathrm{Ti}$ and Ti-(epNPs/HA) ${ }_{4.5}$ were used as controls, where epNPs are empty plasmid-loaded LPSA pNPs, and the preparation of Ti-(epNPs/HA $)_{4.5}$ is the same as Ti-(pNPs/HA $)_{4.5}$ except using empty plasmid. It should be noted that this experiment was stopped at day 4 because thereafter cells overpopulated and apoptosis began. 


\section{Statistical analysis}

Each experiment was performed at least three times. Data were expressed as mean \pm standard deviation. A two-tailed Student's $t$-test was used for statistical analysis. $P<0.05$ is considered statistically significant.

\section{Results and discussion}

To develop a spatiotemporally controllable gene delivery system with high efficiency and safety, we constructed a PEM film functionalized substrate surface via layer-by-layer self-assembly technique. In this PEM film, gene-loaded pNPs were chosen as a cationic polyelectrolyte, and HA was chosen as an anionic polyelectrolyte. For convenient characterization, pEGFP was chosen as a model gene. To initiate self-assembly of PEM films on substrates, large anions were first introduced to substrates via pretreatment, and then pNPs with optimal N/P ratio and HA were sequentially deposited onto surfaces by electrostatic interactions. Thereafter, the properties of PEM films were characterized. Here, because pNPs, developed by our group recently, are a novel degradable gene delivery system with high efficiency and safety in conventional bolus transfection ${ }^{16,17}$ and HA is biodegradable, they are expected to endow PEM film systems with degradability for controlled pDNA release and sustained efficient transfection ability in situ.

\section{Real-time monitoring on buildup of PEM films}

In order to follow construction of PEM films on substrates, we used QCM to monitor the change in frequency and dissipation in real time during self-assembly procedure. Figure 1A shows that within five cycles, the negative of frequency $(-f)$ and the dissipation (D) grow gradually after each deposition, indicating the increase in the mass, thickness, and viscoelasticity of deposited films. The flush of $\mathrm{NaCl}$ slightly reduces $-\mathrm{f}$ and $\mathrm{D}$ because the loosely adhered pNPs leave
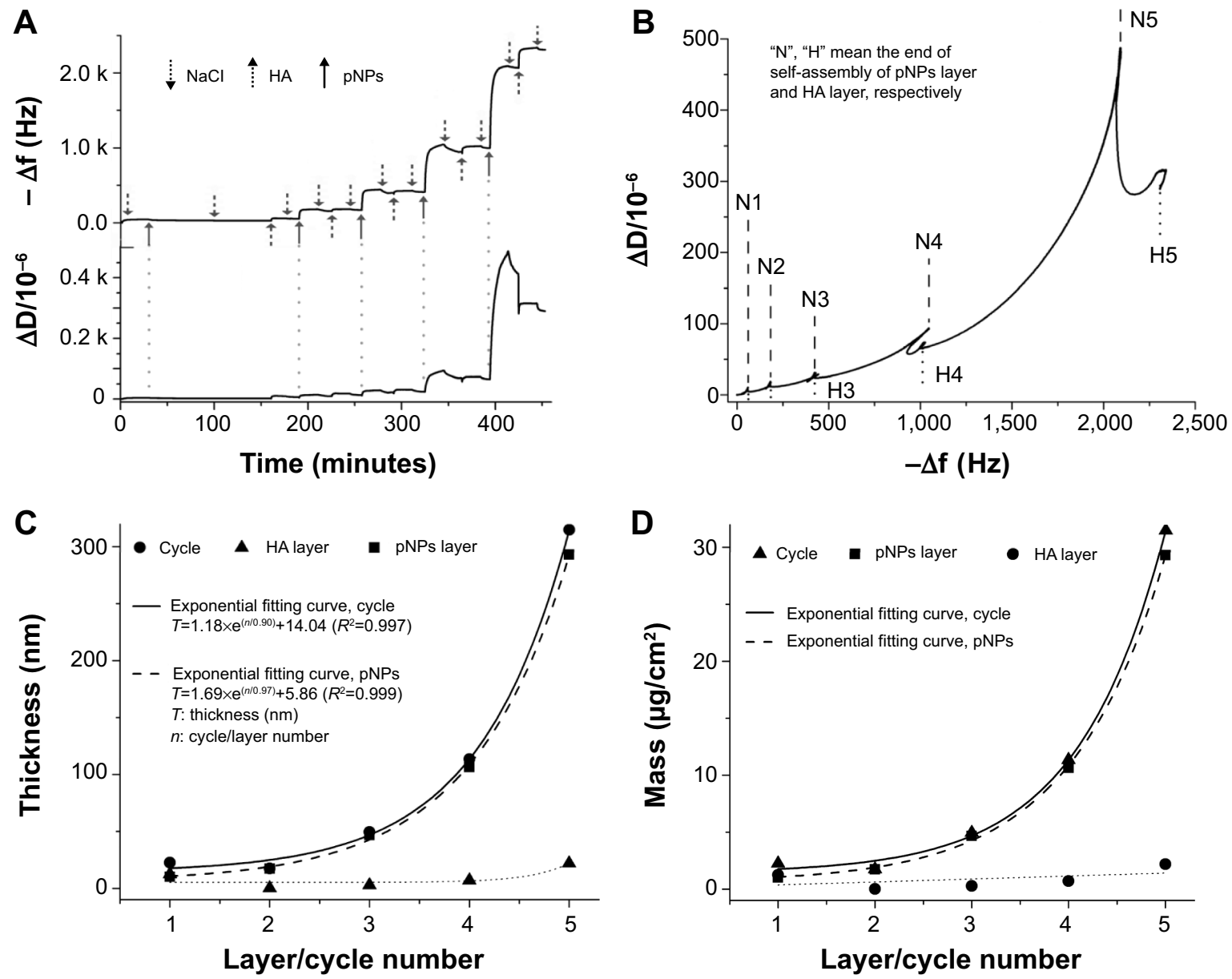

Figure I Analysis on buildup of polyelectrolyte multilayer films by quartz crystal microbalance with dissipation.

Notes: Changes in frequency $(-\Delta f)$ and dissipation $(\Delta D)$ over time $(\mathbf{A})$, time-dependent $\Delta \mathrm{D} / \Delta \mathrm{f}$ data plots $(\mathbf{B})$, hydrodynamic thickness $(\mathbf{C})$, and mass $(\mathbf{D})$ of deposited polyelectrolyte layers as a function of layer/cycle number fitted by the Voigt model. All data were measured with quartz crystal microbalance with dissipation at $15 \mathrm{MHz}$ resonance frequency after successive injection of plasmid deoxyribonucleic acid-loaded lipopolysaccharide-amine nanopolymersomes ( $\mathrm{pNPs}$ ) and hyaluronic acid (HA). 
the layer, suggesting the stability of formed films. It should be noted that within five cycles the film built up regularly, but after that it became unstable and irregular, characterized by the up and down change in frequency, like a seesaw, and the sharp increase in dissipation until overflow. This may imply the change in structure of pNPs: eg, the rupture, rearrangement, and fusion of pNPs after five cycles. Therefore, we only studied the films within five cycles.

Figure $1 \mathrm{~B}$ shows the time-dependent plot of $\Delta \mathrm{D} / \Delta \mathrm{f}$ within five cycles. Each cycle exhibits a similar growth pattern. That is, $\Delta \mathrm{D}$ first increases with $-\Delta \mathrm{f}$ as pNPs deposit (positive slope), then decreases with $-\Delta \mathrm{f}$ as HA deposits (negative slope). Such a change pattern may denote that the film has the same structure, ${ }^{25}$ and HA may diffuse into the interior of the film and crosslink it through electrostatic and hydrogen bonding, thus leading to the decrease in its viscoelasticity.

Normally, there are two growth types for PEM films. One is linear and the other is exponential. To explore the growth type of PEM films formed from pNPs and HA, we fitted their thickness and mass using the Voigt model because they are viscoelastic polymer films. ${ }^{19-22}$ As shown in Figure $1 \mathrm{C}$, the hydrodynamic thickness of films increases exponentially with pNP layer/cycle number, suggesting their exponential growth mode $\left(R^{2}>0.99\right)$. This confirms that there exists polyelectrolyte diffusion between layers. ${ }^{10}$ Such diffusion crosslinks films through hydrogen-bonding and electrostatic interactions, and accordingly enhances the stability of films. The exponential growth allows a large increase in mass within limited assembly cycles, avoiding tedious and time-consuming labor, to obtain enough drug amount compared with linear growth, and exhibits huge potential in drug delivery. The relationship between fitted mass and pNP layer/cycle number is presented in Figure 1D. Similarly, the mass of PEM films grows in an exponential mode and it can be controlled by adjusting the layer number. The mass of pNPs is estimated as 1.01 and $29.30 \mu \mathrm{g} / \mathrm{cm}^{2}$ for layer 1 and 5 , respectively, proving the successful control over loaded pDNA in a relatively wide range in PEM films. Additionally, pNP layer makes a main contribution to film mass (mass ratio, $\mathrm{m}_{\mathrm{pNPs}}: \mathrm{m}_{\mathrm{HA}}=10.67: 1$ in the film with five-cycle deposition), which may be due to their different existence form during assembly (pNPs are an aggregate and HA is a single macromolecule). In this film, HA may act as a threadlike adhesive to pNPs. We believe such composing of PEM film is beneficial for implant materials because unwanted side effects can be avoided by introducing minimum excipients.

\section{Characterization of PEM films}

The formation of PEM films on substrate surfaces and their properties were characterized by XPS, FTIR, water contact angle, zeta potential, and AFM. Their chemical composition was first assayed by XPS. Figure 2A shows the XPS survey spectra and their surface elemental percentage of Ti after different treatments. In the alkaline heat-treated $\mathrm{Ti}(\mathrm{Ti}-\mathrm{OH})$, the coexistence of $\mathrm{Ti}, \mathrm{O}$, and $\mathrm{Na}$ signals means the formation of sodium titanate hydrogel layer, and $\mathrm{C}$ signal is due to the hydrocarbon contamination. In pNP-deposited Ti (Ti-pNPs), the decrease in $\mathrm{Na}$ and $\mathrm{Ti}$ signals and the increase in $\mathrm{C}$ signal combined with the appearance of $\mathrm{N}$ and trace $\mathrm{P}$ signals suggest the presence of NPs and pDNA. The trace $\mathrm{P}$ is because 1) the amount of pDNA in pNPs $(\mathrm{N} / \mathrm{P}=60)$ is small, and 2) the analysis depth of XPS is about $0.5-8 \mathrm{~nm}$, but pDNA exists at the membrane of pNPs whose dry diameter is $\sim 70 \pm 19 \mathrm{~nm}$, and the membrane is covered by the corona of polyethyleneimine (PEI) chain. ${ }^{16}$ As HA has a similar chemical elemental composition to pNPs (both abundant in
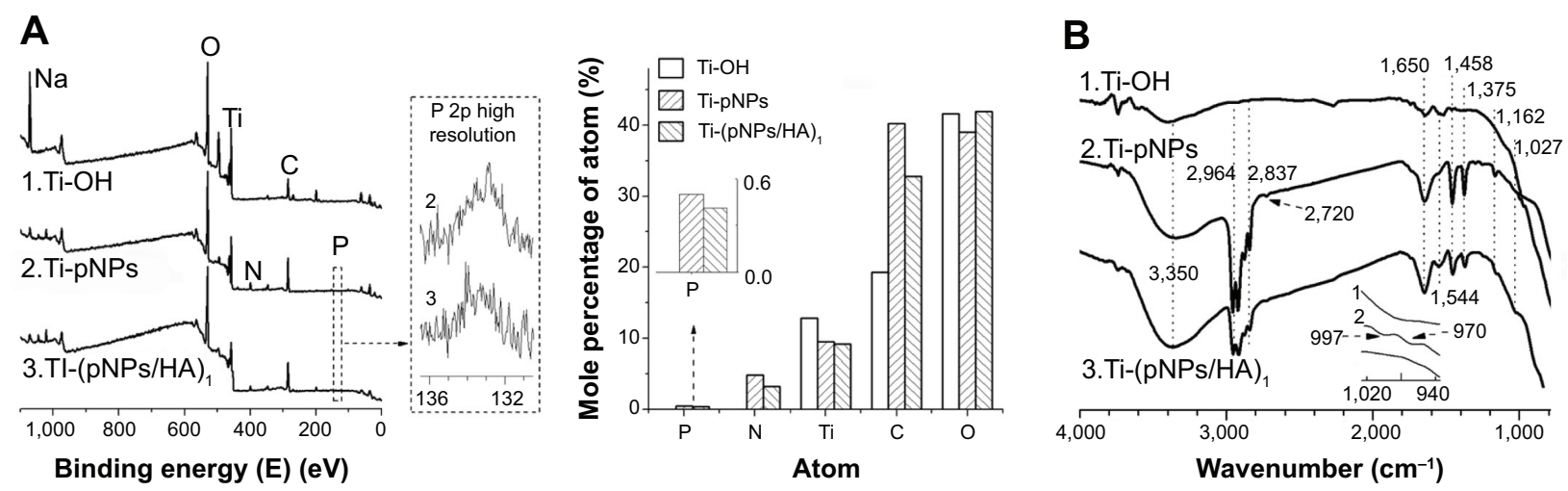

Figure 2 Characterization of polyelectrolyte multilayer films self assembled on titanium.

Notes: X-ray photoelectron spectroscopy survey spectra and their surface elemental percentage (A), Fourier transform infrared spectra (B) of titanium (Ti) treated by alkaline heat, plasmid deoxyribonucleic acid-loaded lipopolysaccharide-amine nanopolymersome (pNP) deposition, and successive deposition of pNPs/hyaluronic acid (HA), respectively.

Abbreviation: P 2p, 2p electron of P element. 
$\mathrm{C}, \mathrm{N}$, and $\mathrm{O}$ elements), no significant changes in element signals are observed after HA deposition ( $\mathrm{Ti}-[\mathrm{pNPs} / \mathrm{HA}]_{1}$ ). It is worth noting that $\mathrm{Ti}$ signal exists in all samples, indicating the PEM film is not so dense and cannot completely cover the substrate. This is reasonable because 1) pNPs are cationic spherical nanovesicles ${ }^{16}$ and the electrostatic repulsion between particles leads to their separation from each other on Ti surface, and 2) as an anionic semiflexible molecule, HA is adsorbed onto cationic pNPs by ion attraction. Therefore, HA layer cannot completely cover the substrate. Such structure is confirmed in AFM images, which will be discussed in detail later.

Figure 2B shows the FTIR spectra (neat, $\mathrm{cm}^{-1}$ ) of treated Ti. After alkaline heat treatment $(\mathrm{Ti}-\mathrm{OH})$, the strong characteristic peak from - $\mathrm{OH}\left(v_{\mathrm{O}-\mathrm{H}}, \sim 3,400 \mathrm{~cm}^{-1}\right)$ is detected, indicating the formation of Ti-OH and the successful introduction of large anions. ${ }^{18,26}$ After pNP treatment (Ti-pNPs), we detected the peaks from pNPs such as amine and amine salts in PEI block: $\sim 3,350 \mathrm{~cm}^{-1}\left(v_{\mathrm{N}-\mathrm{H}}\right.$ in $-\mathrm{NH}_{2}$ and $-\mathrm{NH}-, v_{\mathrm{NH} 3}{ }^{+}, v_{\mathrm{O}-\mathrm{H}}$ in hydroxyls of alginate and Ti-OH), $2,720 \mathrm{~cm}^{-1}\left(\delta_{\mathrm{NH} 3}{ }^{+}, v_{\mathrm{NH} 2}{ }^{+}\right.$, $1,650 \mathrm{~cm}^{-1}\left(\delta_{\mathrm{N}-\mathrm{H}}\right.$ in $-\mathrm{NH}_{2}$ and $-\mathrm{NH}-$, a little contribution from $v_{\mathrm{C}=\mathrm{O}}$ in amide $), 1,544 \mathrm{~cm}^{-1}\left(\delta_{\mathrm{NH} 3}{ }^{+}\right.$, a little from $\delta_{\mathrm{N}-\mathrm{H}}$ in secondary amide), and $1,162 \mathrm{~cm}^{-1}\left(v_{\mathrm{C}-\mathrm{N}}\right.$ in $\left.-\mathrm{NH}_{2}\right)$; alkyls in PEI and cholesterol blocks: $2,964 \mathrm{~cm}^{-1}$ and $2,873 \mathrm{~cm}^{-1}\left(v_{\mathrm{C}-\mathrm{H}}\right.$ in $\left.\mathrm{CH}_{3}\right)$, $2,919 \mathrm{~cm}^{-1}$ and $2,837 \mathrm{~cm}^{-1}\left(v_{\mathrm{C}-\mathrm{H}}\right.$ in $\left.\mathrm{CH}_{2}\right), 1,458 \mathrm{~cm}^{-1}\left(\delta_{\mathrm{C}-\mathrm{H}}\right.$ in $>\mathrm{CH}_{2}$ and $\left.-\mathrm{CH}_{3}\right), 1,375 \mathrm{~cm}^{-1}\left(\delta_{\mathrm{sC}-\mathrm{H}}\right.$ in $\left.\mathrm{CH}_{3}\right)$; and phosphate in pDNA: $997 \mathrm{~cm}^{-1}$ ( $v_{\text {asO-P_o }}$ in phosphate), $970 \mathrm{~cm}^{-1}$ (sugar phosphate-coupled vibration). ${ }^{12,27}$ All of these suggest the successful introduction of pNPs on Ti. After HA treatment (Ti-[pNPs/HA $]_{1}$ ), the spectrum of Ti-(pNPs/HA) is similar

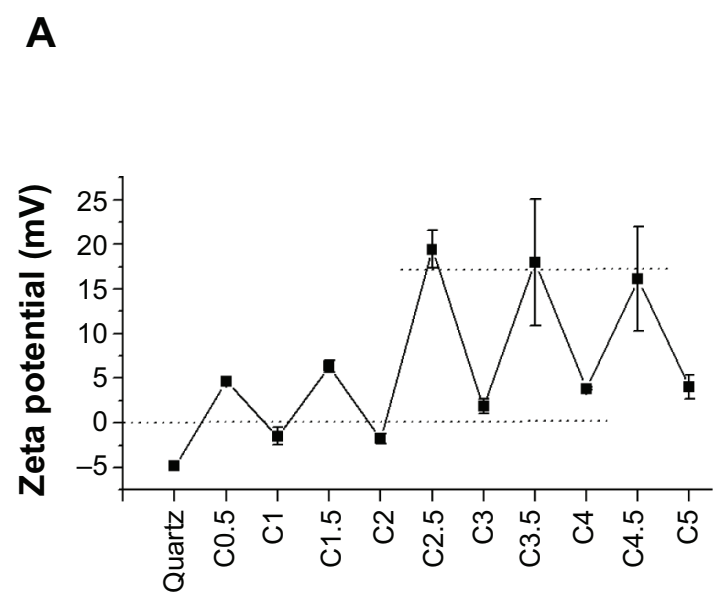

Surface to that of Ti-pNPs, which is ascribed to the similar chemical structure (such as alkyls, amide, carboxyls, hydroxyls) of HA to NPs and noncompact film formation. Despite these, some changes can still be observed, such as the disappearance of pDNA signals, the appearance of $v_{\mathrm{C}-\mathrm{OH}}$ from HA at $1,027 \mathrm{~cm}^{-1}$, and the dropping in amine, amine salt, and alkyl signals. Taken together, all of these results suggest that $\mathrm{pNP}$ layer and HA layer can be introduced to Ti surface one-byone via electrostatic interactions.

The changes in surface zeta potential and contact angle of substrates during self-assembly are interesting (Figure 3A). With self-assembly, the surface zeta potential increases in a zigzag pattern. For quartz surface, it is $-4.83 \mathrm{mV}$, suggesting the introduction of large anions. After pNP treatment $\left(\mathrm{C}_{0.5}\right)$, it increases to $4.62 \mathrm{mV}$, suggesting the introduction of cationic pNPs. Within five cycles, for films whose outmost layer is pNPs $\left(\mathrm{C}_{\mathrm{n}-0.5}, n\right.$ refers to the cycle number $)$, the zeta potential rises from $4.62 \mathrm{mV}$ to $18 \mathrm{mV}$ and then stabilizes there. For films whose outmost layer is $\mathrm{HA}\left(\mathrm{C}_{\mathrm{n}}\right)$, it rises from $-1.51 \mathrm{mV}$ to $8.94 \mathrm{mV}$. It is worth noting that when $\mathrm{n}<2$, the zeta potential of pNP layer $\left(\mathrm{C}_{\mathrm{n}-0.5}\right)$ is much lower than that of free pNPs in solution $(\sim 20 \mathrm{mV})$, but when $n \geq 2$, it gets close. Theoretically, anionic HA deposition should lead to the negative zeta potential, but when $n>2$, it is positive. We believe all of these are caused by the nondense film formation in an exponential growth pattern and the increased contribution of pNPs to the film mass compared with HA (Figure 1D). The nondense film signifies the concomitance of negative and positive charges on each surface. When $\mathrm{n}>2$, the exponential growth leads to a large deposition of

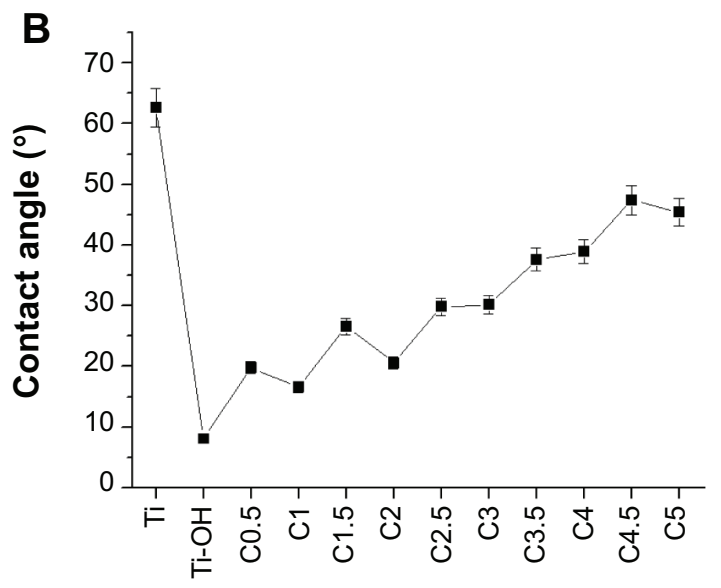

Surface

Figure 3 Surface characterization of polyelectrolyte-multilayer-film-treated substrates.

Notes: Zeta potential $(\mathbf{A})$ and water contact angle $(\mathbf{B})$ of treated substrate surfaces. $C_{n-0.5}$ and $C_{n}$ refer to films whose outmost layer are plasmid deoxyribonucleic acid-loaded lipopolysaccharide-amine nanopolymersomes and hyaluronic acid respectively, where $n$ is cycle number. (A) The dotted lines below and upper refer to the zeta potential of 0 and $18 \mathrm{mV}$, respectively.

Abbreviation: $\mathrm{Ti}$, titanium. 
pNPs, namely large cations on substrates, which brings about sharp elevation of zeta potential until reaching the maximum $(\sim 20 \mathrm{mV})$. Meanwhile, large pNP depositions weaken the negative effects from the small quantity of deposited HA and renders the zeta potential of HA layer positive. The positive charges on surfaces are beneficial for cell attachment because cell membrane possesses negative charges.

Figure $3 \mathrm{~B}$ presents the contact angles of different surfaces. For prime Ti, it is $62.6^{\circ} \pm 4.9^{\circ}$. After alkaline heat treatment (Ti-OH), it decreases to $8.1^{\circ} \pm 2.2^{\circ}$ due to the formation of highly hydrophilic titanate hydrogel layer. With alternately introducing pNPs and HA layer, the contact angle rises in a sawtooth mode at a gradually decreased rate until it reaches $49^{\circ}$ at the fifth cycle. The changes in contact angel indicate that the introduction of polyelectrolyte decreases the surface hydrophilicity, which is attributed to the relative hydrophobicity of pNPs and their interpolyelectrolyte complexes with HA on interfaces. Because of noncompactability of films, with polyelectrolyte deposition, the area of uncovered substrate becomes less and less, and the surface contact angle becomes bigger and bigger until it is basically stable at about $49^{\circ}$. Taken together, the results of XPS, FTIR, zeta potential, and contact angle further confirm the successful self-assembly of films and their exponential growth mode.

\section{AFM observation}

As shown in Figure 4, AFM images reveal that for the first pNP layer (Ti-pNPs), spherical pNPs with positive charges

A

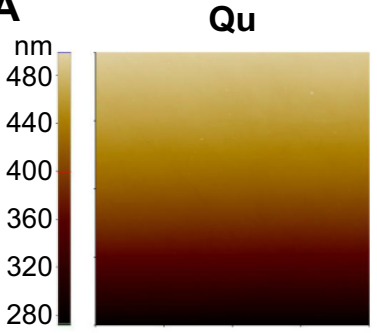

$10 \mu \mathrm{m}$

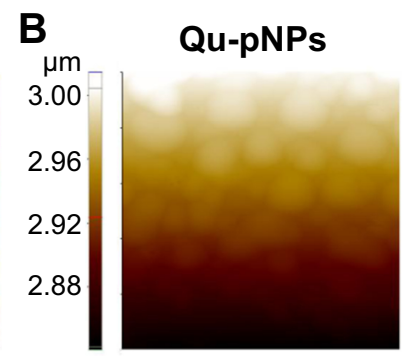

$500 \mathrm{~nm}$

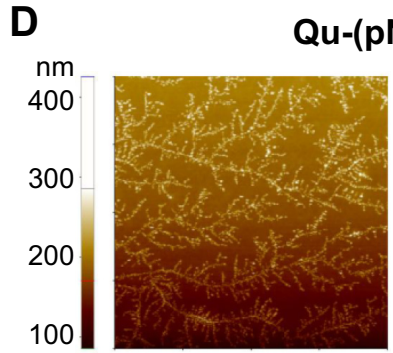

$40 \mu \mathrm{m}$

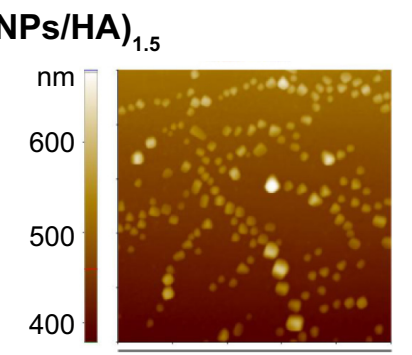

$5 \mu \mathrm{m}$
$20 \mu \mathrm{m}$

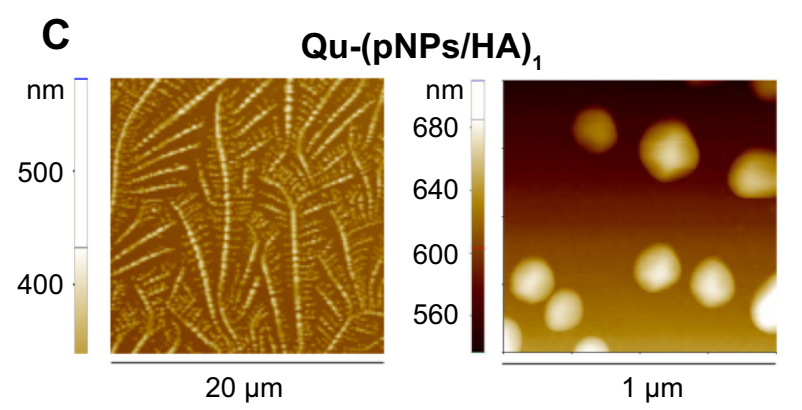

adhere to the substrate with negative charges due to the electrostatic attraction, and they distribute in a separate and uniform pattern because of the electrostatic repulsion among cationic pNPs. After HA deposition (Ti-[pNPs/HA $]_{1}$ ), due to the semiflexible chain nature of HA and complicated electrostatic interactions among HA and pNPs (including repulsion between same kind of charges and attraction between opposite charges), pNPs rearrange and a uniform tree-like structure is formed, whose branches are composed of beaded chains. That is, pNP beads are strung on HA molecular chains. It seems that $\mathrm{Ti}-(\mathrm{pNPs} / \mathrm{HA})_{1}$ acts as a template for subsequent film growth. With self-assembly, the branches become denser and stronger, and finally a three-dimensional nanostructure with jagged, interlocking pNPs is constructed. When cycle number $>2$, Ti-(pNPs/HA) $)_{\mathrm{n}-0.5}$ has a similar structure to $\mathrm{Ti}-(\mathrm{pNPs} / \mathrm{HA})_{\mathrm{n}}$ (data not shown), which may be caused by the small deposited mass of HA, where HA may act as thread-like adhesives for pNPs. In PEM films, no distinct interface between layers is observed, proving the diffusion of polyelectrolytes throughout the films. Visually, the polyelectrolyte films are uniform but not compact, which is consistent with aforementioned XPS data, where the signal of Ti element exists in all modified surfaces.

With polyelectrolyte deposition, the root mean square roughness of films gradually increases from $46 \mathrm{~nm}$ for Ti-pNPs to $119 \mathrm{~nm}$ for Ti-(pNPs/HA) ${ }_{4.5}$. Similarly, Ti-(pNPs/HA) ${ }_{5}$ has almost the same roughness $(122 \mathrm{~nm})$ as Ti-(pNPs/HA) ${ }_{4.5}$, indicating that HA deposition contributes little to film roughness.

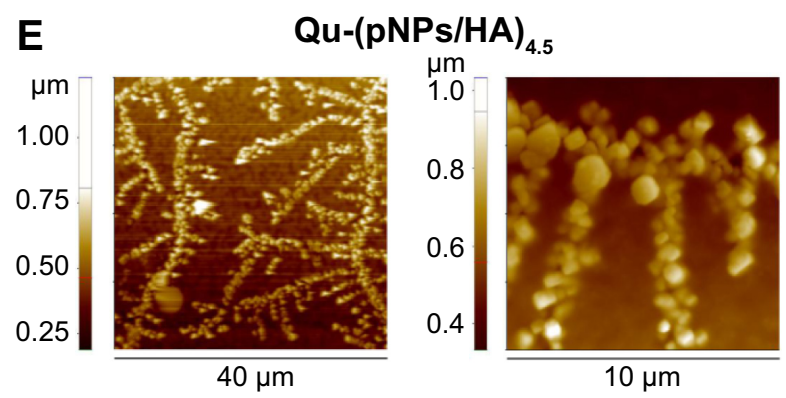

Figure 4 Topography of polyelectrolyte multilayer film functionalized quartz surfaces observed by atomic force microscopy. Notes: (A) Qu. (B) Qu-pNPs. (C) Qu-(pNPs/HA),. (D) Qu-(pNPs/HA) $)_{1.5}$ (E) Qu-(pNPs/HA) ${ }_{4.5}$.

Abbreviations: Qu, quartz; pNPs, plasmid deoxyribonucleic acid-loaded lipopolysaccharide-amine nanopolymersomes; HA, hyaluronic acid. 
It is reported that rough surface is beneficial to cell adhesion and growth, ${ }^{10,28,29}$ which will accordingly improve the substrate-mediated gene transfection because the gene has more chances to contact with cells. Additionally, it is worth noting that in PEM films, pNPs still keep an intact structure (without rupture), suggesting the robustness of pNPs. The structural integrity of $\mathrm{pNPs}$ is very important for pDNA protection and efficient gene transfection.

\section{Release of pDNA from PEM films}

For substrate-mediated gene delivery strategy, to transfect cells surrounding implants, pDNA should be released continuously from the films. We examined the pDNA release from Ti-(pNPs/HA) $)_{4.5}$ incubated in $\mathrm{PBS}$ at $37^{\circ} \mathrm{C}$ for 14 days (Figure 5A). The release profile reveals that pDNA can be released gradually over 14 days. During the first 8 hours, a burst release is observed, which may be from the quick release of loosely adsorbed or inlaid pNPs in films. Then, pDNA releases at a relatively slow and stable rate, and $\sim 74 \%$ of pDNA was released in 14 days. We believe such release pattern is beneficial for therapy because the initial burst release makes the local concentration of pDNA quickly reach a relatively high value. Thus, cells can be efficiently transfected as soon as possible to produce target protein around implants, and then pDNA was released slowly to maintain a long-term drug effect.

The existence form of released pDNA plays a pivotal role in subsequent gene transfection. It is believed that complex form is beneficial to transfection. To explore the existence form of released pDNA, we used gel electrophoresis to analyze the supernatants with or without heparin-dissociation

treatment. As shown in Figure 5B, no DNA band appears in gel electrophoresis when using the supernatant itself collected on day 14 or day 7 postrelease (lane 1 and lane 2), suggesting no free naked pDNA exists. But after being incubated with $20 \mathrm{mg} / \mathrm{mL}$ of heparin for 2 hours (a condition that definitely dissociates pDNA from $\mathrm{pNPs}),{ }^{17}$ strong pDNA bands are observed (lane 3 and lane 4), denoting the existence of free naked pDNA. Therefore, gel electrophoresis results indicate pDNA can be released from PEM films, and the released pDNA in supernatants exists in a complex form. We have demonstrated that $\mathrm{pNPs}$ with optimal N/P ratio are able to overcome a series of barriers during gene delivery and finally obtain $>95 \%$ transfection efficiency in bolus transfection. ${ }^{16}$ Thus, the continuously released pDNA in a complex form (pNPs) provides the possibility for persistent efficient transfection.

\section{Adhesion and proliferation of bone marrow MSCs on PEM-deposited Ti substrates}

The cell response to functionalized implants is very important for later tissue integration and gene transfection, which determines the probability of future clinic application. We used AlamarBlue method and optical microscopy observation to evaluate cell responses. Figure 6A shows that in 15 minutes, the number of MSCs adhered to $\mathrm{Ti}^{-\mathrm{C}_{4.5}}\left(\mathrm{Ti}-[\mathrm{pNPs} / \mathrm{HA}]_{4.5}\right)$ is the maximum, which is 1.6-fold higher than the Ti group. The fluorescent images further confirm the optimal adhesion on Ti- $\mathrm{C}_{4.5}$ among all groups (Figure 6B). Figure $6 \mathrm{C}$ presents the proliferation of MSCs on different Ti surfaces in 5 days. Ti- $\mathrm{C}_{4.5}$ and $\mathrm{Ti}-\mathrm{C}_{5}$ have similar optical density (corresponding

A

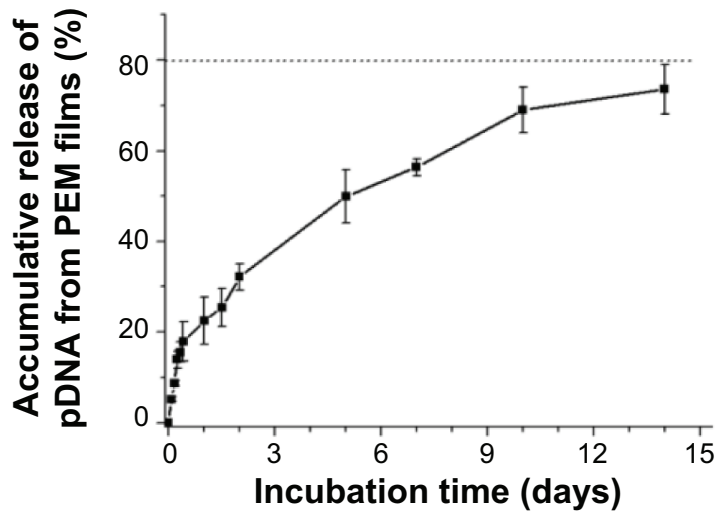

B

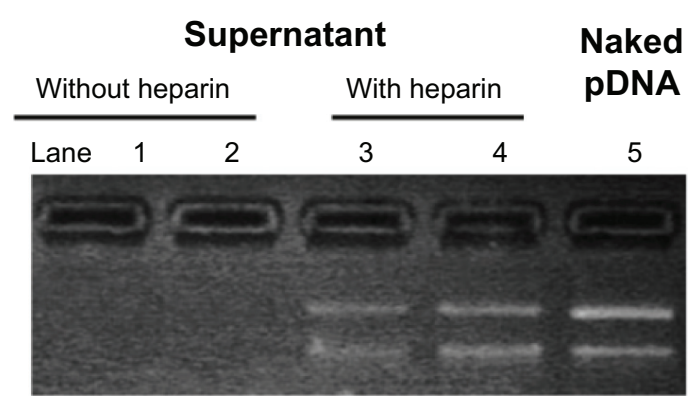

Figure 5 Time-dependent release behavior of pDNA from self assembled films on Ti and their existence form.

Notes: (A) The accumulative release profile of pDNA from Ti-(pNPs/HA) 4.5 incubated in phosphate-buffered saline at $37^{\circ} \mathrm{C}$ for 14 days (measured by ultraviolet-visible spectrophotometer). (B) Gel electrophoresis of naked pDNA (lane 5) and concentrated supernatants without (lane 1, 2) or with (lane 3, 4) heparin-dissociation treatment. Supernatants were collected on the seventh (lane 2, 4) and I4th day (lane I, 3) of release experiment, respectively.

Abbreviations: pDNA, plasmid deoxyribonucleic acid; pNPs, plasmid deoxyribonucleic acid-loaded lipopolysaccharide-amine nanopolymersomes; HA, hyaluronic acid; PEM, polyelectrolyte multilayer; Ti, titanium. 

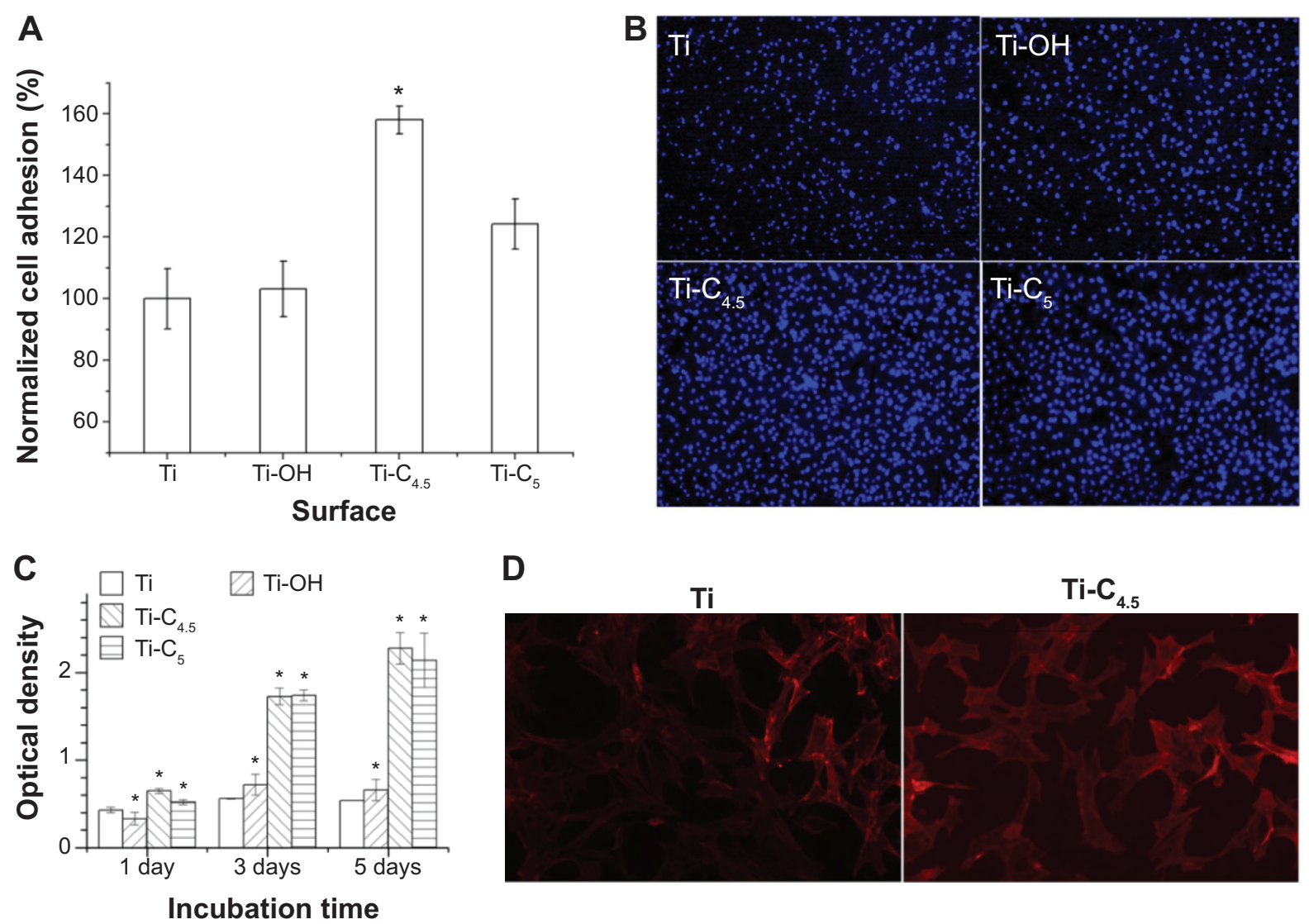

D
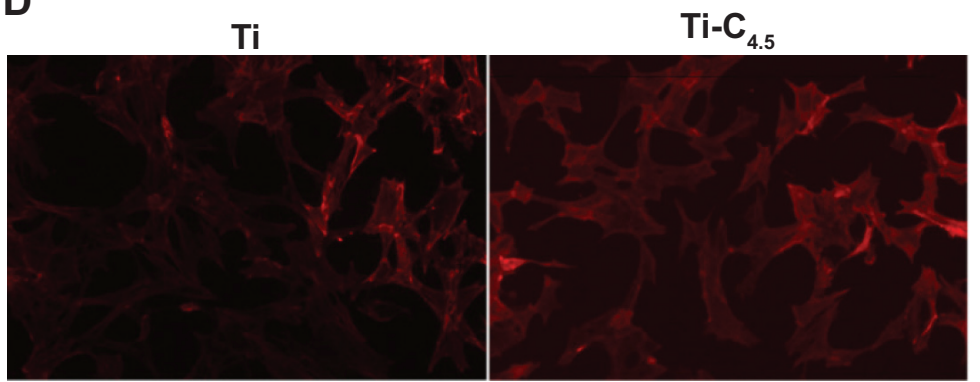

Figure 6 Adhesion and proliferation of MSCs on functionalized Ti surface.

Notes: (A) Adhesion number of MSCs in 15 minutes normalized by Ti group. (B) Fluorescent images of adhered MSCs in 6 hours, whose nuclei are stained by 4',6-diamidino2-phenylindole. (C) Proliferation profile of MSCs for 5 days. (D) Fluorescent images of MSCs cultured for 2 days, whose $\mathrm{F}$-actin is stained by rhodamine phalloidin. Ti- ${ }_{4.5}$ and $\mathrm{Ti}-\mathrm{C}_{5}$ refer to $\mathrm{Ti}-(\mathrm{pNPs} / \mathrm{HA})_{4.5}$ and $\mathrm{Ti}-(\mathrm{pNPs} / \mathrm{HA})_{5} . * \mathrm{P}<0.05$ vs Ti.

Abbreviations: MSCs, mesenchymal stem cells; Ti, titanium; pNPs, plasmid deoxyribonucleic acid-loaded lipopolysaccharide-amine nanopolymersomes; HA, hyaluronic acid; vs, versus.

to cell number), which is much higher than the Ti-OH and Ti groups. Fluorescent images (Figure 6D) show that MSCs spread and grow well on all surfaces at 48 hours. All of these results demonstrate PEM films are noncytotoxic, and their introduction to Ti surface enhances the adhesion and proliferation of MSCs. The positive charges, increased roughness, and suitable hydrophilicity on PEM-modified surfaces may contribute to such enhancement. ${ }^{10,28,29}$ The improvement in adhesion and proliferation of cells on PEM films provide the basis for efficient gene transfection due to more accessible chances for genes to attach to cells.

\section{Transfection in MSCs in vitro}

Even though pNPs can be released gradually from PEM films, the released pDNA may not have the ability to efficiently transfect cells seeded on PEM films. Herein, we evaluated the transfection capability of $\mathrm{Ti}^{-} \mathrm{C}_{4.5}$ in MSCs by using pEGFP as a model gene. Figure 7 shows that at 48 and 96 hours after seeding cells on films, large green fluorescence with uniform distribution is observed on Ti-(pNPs/HA) ${ }_{4.5}$ surface, while no green appears on Ti and Ti-(epNPs/HA $)_{4.5}$ surfaces, suggesting successful transfection in MSCs mediated by Ti-(pNPs/HA) ${ }_{4.5}$ and no detectable autofluorescence caused by transfection. The results of transfection efficiency (percentage of green fluorescence protein-positive cells) determined by flow cytometer further proved this. When using Ti group as a blank reference, for the Ti-(epNPs/HA) ${ }_{4.5}$ group, the transfection efficiency is $\sim 0$, while for the Ti(pNPs/HA) ${ }_{4.5}$ group, it reaches $60.98 \% \pm 14.09 \%$ at 48 hours. At 96 hours, the transfection efficiency slightly decreases to $53.30 \% \pm 10.32 \%$. Such decrease may be caused by the cell proliferation, which is demonstrated by the proliferation experiment (Figure 6). Our system has higher transfection efficiency than those reported substrate-mediated gene delivery systems in MSCs, whose transfection efficiency ranges from $\sim 5 \%$ to $\sim 40 \% \cdot{ }^{3,5,12,15}$ But because of the different experimental conditions, including the quality of plasmid DNA, cell types, cell seeding density, and characterization methods, 

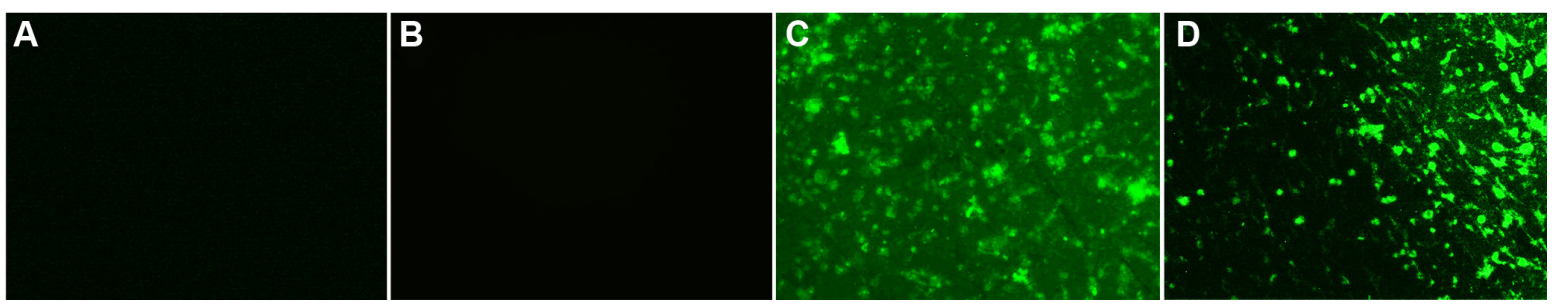

Figure 7 In vitro transfection of MSCs mediated by PEM film-coated Ti for different time periods.

Notes: (A) Ti, 2 days, (B) Ti-(epNPs/HA) $4.5,2$ days, (C) Ti-(pNPs/HA) $)_{4.5}, 2$ days, and (D) Ti-(pNPs/HA) ${ }_{4.5}, 4$ days, respectively.

Abbreviations: MSCs, mesenchymal stem cells; PEM, polyelectrolyte multilayer; Ti, titanium; epNPs, empty plasmid-loaded nanopolymersomes; pNPs, plasmid deoxyribonucleic acid-loaded lipopolysaccharide-amine nanopolymersomes; HA, hyaluronic acid.

it is unscientific to compare these results. Considering that primary body cells (especially stem cells) are more difficult to be transfected compared with cancerous cells, our results may be more meaningful for future clinical applications than those systems performing transfection in cancerous cells, especially in regenerative medicine. These preliminary results about efficient and persistent transfection of our substrate-mediated gene delivery system motivate us to perform detailed and further in vitro and in vivo study using a therapeutic gene in the future.

\section{Conclusion}

We have successfully constructed a PEM film with a threedimensional tree-like nanoarchitecture on substrates by layerby-layer self-assembly technique, where pDNA-loaded pNPs with optimal N/P ratio are used as a polycation and HA as a polyanion. This PEM film grows in an exponential mode, and the incorporated pNPs still keep their nanopolymersome structure. The mass of pNPs in PEM films can be controlled in a relatively wide range via adjusting the self-assembly layers, and therein incorporated pDNA can be gradually released in the complex form over 14 days. Such PEM film-functionalized Ti shows improvement in adhesion and proliferation of MSCs compared with bare Ti and can efficiently and persistently transfect MSCs. Our results suggest that such a system can provide spatial and temporal control over gene transfection through changing PEM film construction parameters, and is promising in localized gene therapy for implantology and cancer therapy. Future work will focus on the controllability of ( $\mathrm{pNPs} / \mathrm{HA})_{n}$ over $\mathrm{pDNA}$ release and their in vitro and in vivo transfection behavior through changing construction parameters such as concentration of polyelectrolyte solutions, layer number, N/P ratio in pNPs, and chemical crosslinking.

\section{Acknowledgments}

The authors would like to thank Guangzhao Zhang and Chunfeng Ma at South China University of Technology for their help in the QCM study. This work was supported by the National Science Foundation of China (81101153, 81371665), and the Science and Technology Plan Funds of Guangdong (2013B051000017).

\section{Disclosure}

The authors report no conflicts of interest in this work.

\section{References}

1. Li K, Feng L, Shen J, et al. Patterned substrates of nano-graphene oxide mediating highly localized and efficient gene delivery. ACS Appl Mater Interfaces. 2014;6:5900-5907.

2. Holmes CA, Tabrizian M. Substrate-mediated gene delivery from glycol-chitosan/hyaluronic acid polyelectrolyte multilayer films. $A C S$ Appl Mater Interfaces. 2013;5:524-531.

3. Bechler SL, Lynn DM. Characterization of degradable polyelectrolyte multilayers fabricated using DNA and a fluorescently-labeled poly $(\beta$-amino ester): shedding light on the role of the cationic polymer in promoting surface-mediated gene delivery. Biomacromolecules. 2012;13:542-552.

4. Blacklock J, Mao G, Oupicky D, et al. DNA release dynamics from bioreducible layer-by-layer films. Langmuir. 2010;26:8597-8605.

5. Jewell CM, Lynn DM. Multilayered polyelectrolyte assemblies as platforms for the delivery of DNA and other nucleic acid-based therapeutics. Adv Drug Deliver Rev. 2008;60:979-999.

6. Blacklock J, Sievers TK, Handa H, et al. Cross-linked bioreducible layer-by-layer films for increased cell adhesion and transgene expression. J Phys Chem B. 2010;114:5283-5291.

7. Jessel N, Oulad-Abdelghani M, Meyer F, et al. Multiple and timescheduled in situ DNA delivery mediated by $\beta$-cyclodextrin embedded in a polyelectrolyte multilayer. PNAS. 2006;103:8618-8621.

8. Tseng W, Haselton FR, Giorgio TD. Transfection by cationic liposomes using simultaneous single cell measurements of plasmid delivery and transgene expression. J Biol Chem. 1997;272:25641-25647.

9. Meyer F, Dimitrova M, Jedrzejenska J, et al. Relevance of bi-functionalized polyelectrolyte multilayers for cell transfection. Biomaterials. 2008;29:618-624.

10. Detzel CJ, Larkin AL, Rajagopalan P. Polyelectrolyte multilayers in tissue engineering. Tissue Eng Part B Rev. 2011;17:101-113.

11. Meyer F, Ball V, Schaaf P, et al. Polyplex-embedding in polyelectrolyte multilayers for gene delivery. Biochim Biophys Acta. 2006; 1758:419-422.

12. Van Den Beucken JJJP, Vos MRJ, Thune PC, et al. Fabrication, characterization, and biological assessment of multilayered DNA-coatings for biomaterial purposes. Biomaterials. 2006;27:691-701.

13. Ren K, Ji J, Shen J. Tunable DNA release from cross-linked ultrathin DNA/PLL multilayered films. Bioconjugate Chem. 2006;17: $77-83$. 
14. Wang W, Li W, Ma N, et al. Non-viral gene delivery methods. Curr Pharm Biotechnol. 2013;14:46-60.

15. Yamauchi F, Koyamatsu Y, Kato K, et al. Layer-by-layer assembly of cationic lipid and plasmid DNA onto gold surface for stent-assisted gene transfer. Biomaterials. 2006;27:3497-3504.

16. Huang Z, Teng W, Liu L, et al. Efficient cytosolic delivery mediated by polymersomes facilely prepared from a degradable, amphiphilic, and amphoteric copolymer. Nanotechnology. 2013;24:265104.

17. Teng W, Huang Z, Chen Y, et al. pVEGF-loaded lipopolysaccharideamine nanopolymersomes for therapeutic angiogenesis. Nanotechnology. 2014;25:65702.

18. Kim H, Miyaji F, Kokubo T, et al. Preparation of bioactive Ti and its alloys via simple chemical surface treatment. J Biomed Mater Res. 1996;32:409-417.

19. Wu B, Li C, Yang H, et al. Formation of polyelectrolyte multilayers by flexible and semiflexible chains. J Phys Chem B. 2012; 116:3106-3114

20. Ma C, Wu B, Zhang G. Protein-protein resistance investigated by quartz crystal microbalance. Colloids Surf B Biointerfaces. 2013;104:5-10.

21. Liu G, Zhao J, Sun Q, et al. Role of chain interpenetration in layer-by-layer deposition of polyelectrolytes. J Phys Chem B. 2008; 112:3333-3338.
22. Voinova MV, Rodahl M, Jonson M, et al. Viscoelastic acoustic response of layered polymer films at fluid-solid interfaces: continuum mechanics approach. Phys Scripta. 1999;59:391-396.

23. Shi R, Wang J, Huang S, et al. Angiotensin II induces vascular endothelial growth factor synthesis in mesenchymal stem cells. Exp Cell Res. 2009;315:10-15.

24. Bidez III PR, Li S, Macdiarmid A, et al. Polyaniline, an electroactive polymer, supports adhesion and proliferation of cardiac myoblasts. J Biomater Sci Polym Ed. 2006;17:199-212.

25. Molino PJ, Higgins MJ, Innis PC, et al. Fibronectin and bovine serum albumin adsorption and conformational dynamics on inherently conducting polymers: a QCM-D study. Langmuir. 2012;28:8433-8445.

26. Kim H, Himeno T, Kawashita M, et al. Surface potential change in bioactive titanium metal during the process of apatite formation in simulated body fluid. J Biomed Mater Res. 2003;67A:1305-1309.

27. Choosakoonkriang S, Lobo BA, Koe GS, et al. Biophysical characterization of PEI/DNA complexes. J Pharm Sci-Us. 2003;92:1710-1721.

28. Bruinink A, Bitar M, Pleskova M, et al. Addition of nanoscaled bioinspired surface features: a revolution for bone-related implants and scaffolds? J Biomed Mater Res Part A. 2014;102A:275-294.

29. Greenbaum E. Thin films and coatings in biology. In: Nazarpour S, editor. Thin Films and Coatings in Biology: Springer; 2013:367.
International Journal of Nanomedicine

\section{Publish your work in this journal}

The International Journal of Nanomedicine is an international, peerreviewed journal focusing on the application of nanotechnology in diagnostics, therapeutics, and drug delivery systems throughout the biomedical field. This journal is indexed on PubMed Central, MedLine, CAS, SciSearch ${ }^{\circledR}$, Current Contents ${ }^{\circledR} /$ Clinical Medicine,

\section{Dovepress}

Journal Citation Reports/Science Edition, EMBase, Scopus and the Elsevier Bibliographic databases. The manuscript management system is completely online and includes a very quick and fair peer-review system, which is all easy to use. Visit http://www.dovepress.com/ testimonials.php to read real quotes from published authors. 